International Journal of Pure and Applied Mathematics

Volume 109 No. 2 2016, 223-232

ISSN: 1311-8080 (printed version); ISSN: 1314-3395 (on-line version)

url: http://www.ijpam.eu

doi: 10.12732/ijpam.v109i2.5

ijpam.eu

\title{
MODIFIED NEW SIXTH-ORDER FIXED POINT ITERATIVE METHODS FOR SOLVING NONLINEAR FUNCTIONAL EQUATIONS
}

\author{
Waqas Nazeer ${ }^{1}$, Muhmmad Tanveer ${ }^{2}$, Kashif Rehman ${ }^{3}$, Shin Min Kang ${ }^{4} \S$ \\ ${ }^{1}$ Division of Science and Technology \\ University of Education \\ Lahore 54000, PAKISTAN \\ ${ }^{2}$ Department of Mathematics and Statistics \\ The University of Lahore \\ Lahore 54000, PAKISTAN \\ ${ }^{3}$ Department of Mathematics \\ Lahore Leads University \\ Lahore 54810, PAKISTAN \\ ${ }^{4}$ Department of Mathematics and RINS \\ Gyeongsang National University \\ Jinju 52828, KOREA
}

\begin{abstract}
In this paper, we present a modified new sixth-order fixed point iterative method for solving nonlinear functional equations and analyzed. The modified new sixth-order fixed point iterative method has convergence of order six and efficiency index 1.8171 which is larger than most of the existing methods and the methods discussed in Table 1. The modified new sixth-order fixed point iterative method converges faster than the methods discussed in Tables 1-6. The comparison tables demonstrate the faster convergence of the modified new sixth-order fixed point method.
\end{abstract}

AMS Subject Classification: 65H05, 65D32

Key Words: nonlinear equation, modified new sixth-order fixed point method, fixed point method, new second order iterative method

Received: April 7, 2015

Published: September 8, 2016

${ }^{\S}$ Correspondence author
(C) 2016 Academic Publications, Ltd. url: www.acadpubl.eu 


\section{Introduction}

The problem, to recall, is solving equations in one variable. We are given a function $f$ and would like to find atleast one solution of the equation $f(x)=0$. Note that, we do not put any restrictions on the function $f$; we need to be able to evaluate the function; otherwise, we cannot even check that a given $x=\alpha$ is true, that is $f(r)=0$. In reality, the mere ability to be able to evaluate the function does not suffice. We need to assume some kind of "good behavior". The more we assume,the more potential we have, on the one hand, to develop fast iteration scheme for finding the root. At the same time, the more we assume, the fewer the functions are going to satisfy our assumptions! This a fundamental paradigm in numerical analysis.

We know that one of the fundamental algorithm for solving nonlinear equations is so-called fixed point iteration method [15].

In the fixed-point iteration method for solving nonlinear equation $f(x)=0$, the equation is usually rewritten as

$$
x=g(x),
$$

where

(i) there exists $[a, b]$ such that $g(x) \in[a, b]$ for all $x \in[a, b]$,

(ii) there exists $[a, b]$ such that $\left|g^{\prime}(x)\right| \leq L<1$ for all $x \in[a, b]$.

Considering the following iteration scheme

$$
x_{n+1}=g\left(x_{n}\right), \quad n=0,1,2 \ldots,
$$

and starting with a suitable initial approximation $x_{0}$, we built up a sequence of approximations, say $\left\{x_{n}\right\}$, for the solution of nonlinear equation, say $\alpha$. the scheme will be converge to $\alpha$, provided that

(i) the initial approximation $x_{0}$ is chosen in the interval $[a, b]$,

(ii) $\left|g^{\prime}(x)\right|<1$ for all $x \in[a, b]$,

(iii) $a \leq g(x) \leq b$ for all $x \in[a, b]$.

It is well known that the fixed point method has first order convergence.

Kang et al. [18] described a new second order iterative method for solving nonlinear equations extracted from fixed point method by following the approach of [7] as follows:

If $g^{\prime}(x) \neq 1$, we can modify (1.1) by adding $\theta \neq-1$ to both sides as:

$$
\theta x+x=(1+\theta) x=\theta x+g(x),
$$

which implies that

$$
x=\frac{\theta x+g(x)}{1+\theta}=g_{\theta}(x) .
$$


In order for $g_{\theta}(x)$ to be efficient, we can choose $\theta$ such that $g_{\theta}^{\prime}(x)=0$, we yields

$$
\theta=-g^{\prime}(x)
$$

so that (1.3) takes the form

$$
x=\frac{-x g^{\prime}(x)+g(x)}{1-g^{\prime}(x)} .
$$

For a given $x_{0}$, we calculate the approximation solution $x_{n+1}$, by the iteration scheme

$$
x_{n+1}=\frac{-x_{n} g^{\prime}\left(x_{n}\right)+g\left(x_{n}\right)}{1-g^{\prime}\left(x_{n}\right)}, \quad g^{\prime}\left(x_{n}\right)=-1 .
$$

This is so-called a new second order iterative method for solving nonlinear equations, which converges quadratically.

To prove (1.4) analytically, we use Taylor expansion, expanding (1.1) about the point $x_{n}$ such that

$$
x=g\left(x_{n}\right)+\left(x-x_{n}\right) g^{\prime}\left(x_{n}\right)+\frac{1}{2 !}\left(x-x_{n}\right)^{2} g^{\prime \prime}\left(x_{n}\right)+\cdots .
$$

If $g^{\prime}\left(x_{n}\right) \neq 0$, we can evaluate the above expression as follows:

$$
g\left(x_{n}\right)+\left(x-x_{n}\right) g^{\prime}\left(x_{n}\right)=x .
$$

If we choose $x_{n+1}$ the root of equation, then we have

$$
x_{n+1}=\frac{-x_{n} g^{\prime}\left(x_{n}\right)+g\left(x_{n}\right)}{1-g^{\prime}\left(x_{n}\right)}, \quad g^{\prime}\left(x_{n}\right)=-1 .
$$

This is the analytical prove of new second order iterative method [18] for solving nonlinear equations, which converges quadratically. From (1.5) one can evaluate

$$
x_{n+1}=\frac{-x_{n} g^{\prime}\left(x_{n}\right)+g\left(x_{n}\right)}{1-g^{\prime}\left(x_{n}\right)}+\frac{\left(g\left(x_{n}\right)-x_{n}\right)^{2}}{\left(1-g^{\prime}\left(x_{n}\right)\right)^{3}} g^{\prime \prime}\left(x_{n}\right), \quad g^{\prime}\left(x_{n}\right)=-1 .
$$

This is so-called the modified new third-order iterative method [5] for non-linear equations, which converges cubically.

During the last century, the numerical techniques for solving nonlinear equations have been successfully applied (see, e.g., [1-4, 6-14, 16, 17, 19-30] and the references therein). McDougall and Wotherspoon [22] modified the Newton's method and their modified Newton's method have convergence of order $1+\sqrt{2}$. 
Theorem 1.1. ([7]) Suppose that $g \in C^{n}[a, b]$. If $g^{k}(x)=0$ for $k=$ $1,2, \ldots, p-1$ and $g^{k}(x) \neq 0$, the sequence $\left\{x_{n}\right\}$ is of order $p$.

In this paper, a modified new sixth-order fixed point iterative method for solving nonlinear functional equations having convergence of order 6 and efficiency index 1.8171 extracted from the modified new third-order iterative method for solving nonlinear equations [5] motivated by the technique of McDougall and Wotherspoon [22] has been presented. The proposed modified new sixth-order fixed point iterative method applied to solve some problems in order to assess its validity and accuracy.

\section{Main Results}

Let $f: X \subset \mathbb{R} \rightarrow \mathbb{R}$ for an open interval $X$ be a scalar function and consider that the nonlinear equation $f(x)=0$ (or $x=g(x)$ ), where $g(x): X \subset \mathbb{R} \rightarrow \mathbb{R}$. Then we have modified new third-order iterative method for nonlinear equations

$$
x_{n+1}=\frac{-x_{n} g^{\prime}\left(x_{n}\right)+g\left(x_{n}\right)}{1-g^{\prime}\left(x_{n}\right)}+\frac{\left(g\left(x_{n}\right)-x_{n}\right)^{2}}{\left(1-g^{\prime}\left(x_{n}\right)\right)^{3}} g^{\prime \prime}\left(x_{n}\right), \quad g^{\prime}\left(x_{n}\right)=-1 .
$$

By following the approach of McDougall and Wotherspoon [22], we develop a modified new sixth-order fixed point iterative method as follows:

Initially, we choose two starting points $x$ and $x^{*}$. Then we set $x^{*}=x$, our modified new sixth-order fixed point iterative method that we examine herein is given by

$$
\begin{aligned}
x_{0}^{*}= & x_{0}, \\
x_{1}= & \frac{-x_{0} g^{\prime}\left(\frac{1}{2}\left[x_{0}+x_{0}^{*}\right]\right)+g\left(x_{0}\right)}{1-g^{\prime}\left(\frac{1}{2}\left[x_{0}+x_{0}^{*}\right]\right)} \\
& +\frac{\left(g\left(x_{0}\right)-x_{0}\right)^{2}}{\left(1-g^{\prime}\left(\frac{1}{2}\left[x_{0}+x_{0}^{*}\right]\right)\right)^{3}} g^{\prime \prime}\left(\frac{1}{2}\left[x_{0}+x_{0}^{*}\right]\right),
\end{aligned}
$$

which implies (for $n \geq 1$ )

$$
\begin{aligned}
x_{n}^{*}= & \frac{-x_{n} g^{\prime}\left(\frac{1}{2}\left[x_{n-1}+x_{n-1}^{*}\right]\right)+g\left(x_{n}\right)}{1-g^{\prime}\left(\frac{1}{2}\left[x_{n-1}+x_{n-1}^{*}\right]\right)} \\
& +\frac{\left(g\left(x_{n}\right)-x_{n}\right)^{2}}{\left(1-g^{\prime}\left(\frac{1}{2}\left[x_{n-1}+x_{n-1}^{*}\right]\right)\right)^{3}} g^{\prime \prime}\left(\frac{1}{2}\left[x_{n-1}+x_{n-1}^{*}\right]\right) .
\end{aligned}
$$




$$
\begin{aligned}
x_{n+1}= & \frac{-x_{n} g^{\prime}\left(\frac{1}{2}\left[x_{n}+x_{n}^{*}\right]\right)+g\left(x_{n}\right)}{1-g^{\prime}\left(\frac{1}{2}\left[x_{n}+x_{n}^{*}\right]\right)} \\
& +\frac{\left(g\left(x_{n}\right)-x_{n}\right)^{2}}{\left(1-g^{\prime}\left(\frac{1}{2}\left[x_{n}+x_{n}^{*}\right]\right)\right)^{3}} g^{\prime \prime}\left(\frac{1}{2}\left[x_{n}+x_{n}^{*}\right]\right),
\end{aligned}
$$

where $g^{\prime}\left(\frac{1}{2}\left[x_{n}+x_{n}^{*}\right]\right) \neq-1$. These are the main steps of our modified new sixth-order fixed point iterative method.

The value of $x_{2}$ is calculated from $x_{1}$ using $g\left(x_{1}\right)$ and the values of first derivative of $g(x)$ evaluated at $\frac{1}{2}\left(x_{1}+x_{1}^{*}\right)$ (which is more appropriate value of the derivative to use than the one at $x_{1}$ ), and this same value of derivative is re-used in the next predictor step to obtain $x_{3}^{*}$. This re-use of the derivative means that the evaluations of the starred values of $x$ in (2.3) essentially come for free, which then enables the more appropriate value of the derivatives to be used in the corrector step (2.4).

\section{Convergence Analysis}

Theorem 3.1. Let $f: X \subset \mathbb{R} \rightarrow \mathbb{R}$ for an open interval $X$ and consider that the nonlinear equation $f(x)=0$ (or $x=g(x)$ ) has a simple root $\alpha \in X$, where $g(x): X \subset \mathbb{R} \rightarrow \mathbb{R}$ be sufficiently smooth in the neighborhood of $\alpha$. Then the convergence order of the modified new sixth-order fixed point iterative method given in (2.4) is at least six.

Proof. To analysis the convergence of the modified new sixth-order fixed point iterative method (2.4), let

$$
\begin{aligned}
H(x)= & \frac{-x g^{\prime}\left(\frac{1}{2}\left[x+x^{*}\right]\right)+g(x)}{1-g^{\prime}\left(\frac{1}{2}\left[x+x^{*}\right]\right)} \\
& +\frac{(g(x)-x)^{2}}{\left(1-g^{\prime}\left(\frac{1}{2}\left[x+x^{*}\right]\right)\right)^{3}} g^{\prime \prime}\left(\frac{1}{2}\left[x+x^{*}\right]\right),
\end{aligned}
$$

where $g^{\prime}\left(\frac{1}{2}\left[x+x^{*}\right]\right) \neq-1$. Let $\alpha$ be a simple zero of $f$ and $f(\alpha)=0$ (or 
$g(\alpha)=\alpha)$. Then we can easily deduce by using the software Maple that

$$
\begin{aligned}
H(\alpha) & =\alpha, \\
H^{\prime}(\alpha) & =0, \\
H^{\prime \prime}(\alpha) & =0, \\
H^{\prime \prime \prime}(\alpha) & =0, \\
H^{(i v)}(\alpha) & =0, \\
H^{(v)}(\alpha) & =0, \\
H^{(v i)}(\alpha) & \neq 0 .
\end{aligned}
$$

Now, since $H^{(v i)}(\alpha) \neq 0$, then according to Theorem 1.1, the modified new sixth-order fixed point iterative method (2.4) has sixth order convergence.

\section{Comparison of Efficiency Indices}

Frontini and Sormani [11], Homeier [14] and Weerakoon and Fernando [30] have presented numerical methods having cubic convergence. In each iteration of these numerical methods three evaluations are required of either the function or its derivative. The best way of comparing these numerical methods is to express the of convergence per function or derivative evaluation, the so-called "efficiency" of the numerical method. On this basis, the Newton's method has an efficiency of $2^{\frac{1}{2}} \approx 1.4142$, the cubic convergence methods have an efficiency of $3^{\frac{1}{3}} \approx 1.4422$.

Kuo [21] has developed several methods that each require two function evaluations and two derivative evaluations and these methods achieve an order of convergence of either five or six, so having efficiencies of $5^{\frac{1}{4}} \approx 1.4953$ and $6^{\frac{1}{4}} \approx 1.5651$, respectively. In these Kuo's methods the denominator is a linear combination of derivatives evaluated at different values of $x$, so that, when the starting value of $x$ is not close to the root, this denominator may go to zero and the methods may not converge. Of the four 6 -th order methods suggested in Kuo [21], if the ratio of function's derivatives at the two value of $x$ differ by a factor of more than three, then the method gives an infinite change in $x$. That is, the derivatives at the predictor and corrector stages can both be the same sign, but if their magnitudes differ by more than a factor of three, the method does not converge.

Jarrat [16] developed a 4-th order method that requires only one function evaluation and two derivative evaluations, and similar 4-th order method have 
been described by Soleymani et al. [27]. Jarrat's method is similar to those of Kuo's methods in that if the ratio of derivatives at the predictor and corrector steps exceeds a factor of three, the method gives an infinite change in $x$. Jarratt's methods is similar to those of Kou in that if the ratio of the derivatives at the predictor and corrector steps exceeds a factor of three, the method gives an infinite change in $x$.

While our modified new sixth-order fixed point iterative method (MNFIM) has an efficiency of $6^{\frac{1}{3}} \approx 1.8171$. The efficiencies of the methods we have discussed are summarized in Table 1 given below.

Table 1. Comparison of efficiencies of various methods

\begin{tabular}{|c|c|c|}
\hline Method & $\begin{array}{c}\text { Number of function or } \\
\text { derivative evaluations }\end{array}$ & Efficiency index \\
\hline Newton, quadratic & 2 & $2^{\frac{1}{2}} \approx 1.4142$ \\
\hline Cubic methods & 3 & $3^{\frac{1}{3}} \approx 1.4422$ \\
\hline Kou's 5-th order & 4 & $5^{\frac{1}{4}} \approx 1.4953$ \\
\hline Kou's 6-th order & 4 & $6^{\frac{1}{4}} \approx 1.5651$ \\
\hline Jarratt's 4-th order & 3 & $4^{\frac{1}{3}} \approx 1.5874$ \\
\hline Secant & 1 & $0.5(1+\sqrt{5}) \approx 1.6180$ \\
\hline MNFIM & 3 & $6^{\frac{1}{3}} \approx 1.8171$ \\
\hline
\end{tabular}

\section{Applications}

In this section we included some nonlinear functions to illustrate the efficiency of our developed modified two-step fixed point iterative method. We compare the modified new sixth-order fixed point iterative method with the fixed point method and the new second order iterative method [18] by choosing following test function,

$$
\begin{aligned}
& f(x)=x+\ln (x-2), \quad g(x)=2+e^{-x}, \\
& f(x)=x^{3}+4 x^{2}-10, \quad g(x)=\sqrt{\frac{10}{4+x}} \\
& f(x)=x^{2}-e^{x}-3 x+2, \quad g(x)=\ln \left(x^{2}-3 x+2\right), \\
& f(x)=x^{3}+x^{2}-3 x-2, \quad g(x)=\left(2+3 x-x^{2}\right)^{\frac{1}{3}}, \\
& f(x)=x^{3}+4 x^{2}+8 x+8, \quad g(x)=-\left(1+\frac{1}{2} x^{2}+\frac{1}{8} x^{3}\right) .
\end{aligned}
$$


Comparison is shown in Tables 2-6.

Table 2. Comparison of FPM, NIM and MNFIM

$\left(f(x)=x+\ln (x-2), g(x)=2+e^{-x}, x_{0}=2.2\right)$

\begin{tabular}{|c|c|c|c|c|}
\hline Method & $N$ & $N_{f}$ & $\left|f\left(x_{n+1}\right)\right|$ & $x_{n+1}$ \\
\hline NIM & 3 & 6 & $2.531488 e-11$ & \\
\hline FPM & 20 & 20 & $7.263444 e-20$ & 2.12002823898764122948 \\
\hline MNFIM & 2 & 6 & $7.636960 e-23$ & \\
\hline
\end{tabular}

Table 3. Comparison of FPM, NIM and MNFIM

$\left(f(x)=x^{3}+4 x^{2}-10, g(x)=\sqrt{\frac{10}{4+x}}, x_{0}=1.5\right)$

\begin{tabular}{|c|c|c|c|c|}
\hline Method & $N$ & $N_{f}$ & $\left|f\left(x_{n+1}\right)\right|$ & $x_{n+1}$ \\
\hline FPM & 22 & 2 & $4.376963 e-20$ & \\
\hline NIM & 4 & 8 & $1.357704 e-40$ & 1.36523001341409684576 \\
\hline MNFIM & 2 & 6 & $4.467745 e-20$ & \\
\hline
\end{tabular}

Table 4. Comparison of FPM, NIM and MNFIM

$\left(f(x)=x^{2}-e^{x}-3 x+2, g(x)=\ln \left(x^{2}-3 x+2\right), x_{0}=0.8\right)$

\begin{tabular}{|c|c|c|c|c|}
\hline Method & $N$ & $N_{f}$ & $\left|f\left(x_{n+1}\right)\right|$ & $x_{n+1}$ \\
\hline FPM & & & Diverged & \\
\hline NIM & 6 & 12 & $2.480682 e-12$ & 0.25753028543986076045 \\
\hline MNFIM & 3 & 9 & $2.202949 e-21$ & \\
\hline
\end{tabular}

Table 5. Comparison of FPM, NIM and MNFIM

$\left(f(x)=x^{3}+x^{2}-3 x-2, g(x)=\left(2+3 x-x^{2}\right)^{\frac{1}{3}}, x_{0}=1\right)$

\begin{tabular}{|c|c|c|c|c|}
\hline Method & $N$ & $N_{f}$ & $\left|f\left(x_{n+1}\right)\right|$ & $x_{n+1}$ \\
\hline FPM & 14 & 14 & $3.533701 e-21$ & \\
\hline NIM & 5 & 10 & $4.056640 e-33$ & 1.61803398874989484820 \\
\hline MNFIM & 3 & 9 & $6.282689 e-20$ & \\
\hline
\end{tabular}

Table 6. Comparison of FPM, NIM and MNFIM

$\left(f(x)=x^{3}+4 x^{2}+8 x+8, g(x)=-\left(1+\frac{1}{2} x^{2}+\frac{1}{8} x^{3}\right), x_{0}=-1.9\right)$

\begin{tabular}{|c|c|c|c|c|}
\hline Method & $N$ & $N_{f}$ & $\left|f\left(x_{n+1}\right)\right|$ & $x_{n+1}$ \\
\hline FPM & 62 & 62 & $9.569772 e-20$ & \\
\hline NIM & 4 & 8 & $1.142208 e-20$ & -2.00000000000000000000 \\
\hline MNFIM & 3 & 9 & 0 & \\
\hline
\end{tabular}

Table 2-6. Shows the numerical comparisons of the modified new sixthorder fixed point iterative method (MNFIM) with the fixed point method (FPM) and the new second order iterative method (NIM) [18]. The columns represent the number of iterations $N$ and the number of functions or derivatives 
evaluations $N_{f}$ required to meet the stopping criteria, and the magnitude $|f(x)|$ of $f(x)$ at the final estimate $x_{n}$.

\section{Conclusions}

A modified new sixth-order iterative method (MNFIM) for solving nonlinear functions has been established. We can concluded from Tables 1-6 that

1. The efficiency index of MNFIM is 1.8171 which is larger than the efficiency index of most of the existing methods and the methods discussed in Table 1.

2. MNFIM has convergence of order six.

3. By using some examples the performance of MNFIM is also discussed. MNFIM is performing very well in comparison to the fixed point method and the new iterative method (1.6) as discussed in Tables 2-6.

\section{References}

[1] S. Abbasbandy, Improving Newton-Raphson method for nonlinear equa- tions by modified Adomian decomposition method, Appl. Math. Comput., 145 (2003), 887-893. doi: 10.1016/S0096-3003(03)00282-0.

[2] M.S. Ahmad, A. Ali, M. Tanveer, A. Aslam, W. Nazeer, New fixed point iterative method for solving nonlinear functional equations, Sci. Int. (Lahore), 27 (2015), 1815-1817.

[3] A. Ali, M.S. Ahmad, W. Nazeer, M. Tanveer, New modified two-step jungck iterative method for solving nonlinear functional equations, Sci. Int. (Lahore), 27 (2015), 29592963.

[4] A. Ali, M.S. Ahmad, M. Tanveer, Q. Mehmood, W. Nazeer, Modified two-step fixed point iterative method for solving nonlinear functional equations, Sci. Int. (Lahore), $\mathbf{2 7}$ (2015), 1737-1739.

[5] A. Ali, Q. Mehmood, M. Tanveer, A. Aslam, W. Nazeer, Modified new third-order iterative method for non-linear equations, Sci. Int. (Lahore), 27 (2015), 1741-1744.

[6] A. Ali, W. Nazeer, M. Tanveer, M. Ahmad, Modified golbabai and javidi's method (MGJM) for solving nonlinear functions with convergence of order six, Sci. Int. (Lahore), 28 (2015), 89-93.

[7] E. Babolian, J. Biazar, On the order of convergence of Adomian method, Appl. Math. Comput., 130 (2002), 383-387. doi: 10.1016/S0096-3003(01)00103-5.

[8] E. Babolian, J. Biazar, Solution of nonlinear equations by modified Adomian decomposition method, Appl. Math. Comput., 132 (2002), 167-172. doi: 10.1016/S00963003(01)00184-9.

[9] R.L. Burden, J.D. Faires, Numerical Analysis (Sixth ed.), Brooks/Cole Publishing Co., California, 1998. 
[10] D. Chen, I.K. Argyros, Q.S. Qian, A note on the Halley method in Banach spaces, Appl. Math. Comput., 58 (1993), 215-224. doi: 10.1016/0096-3003(93)90137-4.

[11] M. Frontini, E. Sormani, Some variant of Newton's method with third- order convergence, Appl. Math. Comput., 140 (2003), 419-426. doi: 10.1016/S0096-3003(02)00238-2.

[12] J. M. Gutiérrez, M. A. Hernández, A family of Chebyshev-Halley type methods in Banach spaces, Bull. Austral. Math. Soc., 55 (1997), 113-130. doi: 10.1017/s0004972700030586.

[13] J.M. Gutiérrez, M.A. Hernández, An acceleration of Newtons method: super-Halley method, Appl. Math. Comput., 117 (2001), 223-239. doi: 10.1016/S0096-3003(99)001757 .

[14] H. Homerier, A modified Newton's Method for root finding with cubic convergence, $J$. Comput. Appl. Math., 157 (2003), 227-230. doi: 10.1016/S0377-0427(03)00391-1.

[15] E. Isaacson and H. B. Keller, Analysis of Numerical Methods, John Wiley \& Sons, New York, USA, 1966.

[16] P. Jarratt, Some efficient fourth order multipoint methods for solving equations, BIT, 9 (1969), 119-124. doi: 10.1007/BF01933248.

[17] S.M. Kang, W. Nazeer, M. Tanveer, Q. Mehmood, K. Rehman, Improvements in NewtonRapshon method for nonlinear equations using modified Adomian decomposition method, Int. J. Math. Anal., 9 (2015), 1919-1928. doi: 10.12988/ijma.2015.54124.

[18] S.M. Kang, A. Rafiq and Y. C. Kwun, A New Second-Order Iteration Method for Solving Nonlinear Equations, Abstr. Appl. Anal., 2013 (2013), Article ID 487062, 4 pages.

[19] S.M. Kang, S.M. Ramay, M. Tanveer, W. Nazeer, Polynomiography via an iterative method corresponding to Simpson's $\frac{1}{3}$ rule, J. Nonlinear Sci. Appl., 9 (2016), 967-976.

[20] M. S. Khan, A. Nazir, W. Nazeer, Iterative method for solving nonlinear functions with convergence of order four, Sci. Int. (Lahore), 28 (2016), 77-81.

[21] J. Kuo, The improvements of modified Newton's method, Appl. Math. Comput., 189 (2007), 602-609. doi: 10.1016/j.amc.2006.11.115.

[22] T.J. McDougall, S.J. Wotherspoon, A simple modification of Newton's method to achieve convergence of order $1+\sqrt{2}$, Appl. Math. Lett., 29 (2014), 20-25. doi: 10.1016/j.aml.2013.10.008.

[23] W. Nazeer, S.M. Kang, M. Tanveer, Modified Abbasbandy's method for solving nonlinear functions with convergence of order six, Int. J. Math. Anal., 9 (2015), 2011-2019. doi: 10.12988/ijma.2015.56166.

[24] W. Nazeer, A. Naseem, S.M. Kang, Y.C. Kwun., Generalized Newton Raphson's method free from second derivative, J. Nonlinear Sci. Appl., 9 (2016), 2823-2831.

[25] W. Nazeer, M. Tanveer, S.M. Kang, A. Naseem, A new Householder's method free from second derivatives for solving nonlinear equations and polynomiography, J. Nonlinear Sci. Appl., 9 (2016), 998-1007.

[26] A. Quarteroni, R. Sacco, F. Saleri, Numerical Mathematics, Springer-Verlag, New York, 2000 .

[27] F. Soleymani, S.K. Khattri, S.K. Vanani, Two new classes of optimal Jarratt-type fourthorder methods, Appl. Math. Lett., 25 (2012), 847-853. doi: 10.1016/j.aml.2011.10.030.

[28] J. Stoer, R. Bulirsch, Introduction to Numerical Analysis (Third ed.), Springer-Verlag, New York, 2002. 
[29] M. Tanveer, M. Ahamd, A. Ali, W. Nazeer, K. Rehman, Modified householder's method (MHHM) for solving nonlinear functions with convergence of order six, Sci. Int. (Lahore), 28 (2016), 83-87.

[30] S. Weerakoon, T.G.I. Fernando, A variant of Newton's method with accelerated third-order convergence, Appl. Math. Lett., 13 (2000), 87-93. doi: 10.1016/S08939659(00)00100-2. 
